\title{
Multiboson Measurements in CMS and ATLAS
}

\section{Saptaparna Bhattacharya* on behalf of the ATLAS and CMS Collaborations}

Department of Physics and Astronomy, Northwestern University, Evanston, IL

E-mail: saptaparna.bhattacharya@northwestern.edu

The collection of more than $150 \mathrm{fb}^{-1}$ of data at the Large Hadron Collider (LHC) allows for the exploration of rare processes predicted in the Standard Model (SM) that were previously inaccessible, such as the production of triple gauge bosons. The first observation of triboson production was announced by the CMS Collaboration in April, 2020 and marks an important achievement in SM physics. The first observation of tribosons (VVV) in leptonic final states is reported. The VVV process is observed (expected) with a significance of 5.7 (5.9) $\sigma$ and the measured signal strength is $1.02_{-0.23}^{+0.26}$. The abundance of data also enables the analyses of diboson processes in considerable detail. Two representative precision analyses are discussed, namely, the measurement of $\mathrm{W}^{+} \mathrm{W}^{-}$using data from both ATLAS and CMS experiments and the detailed study of the $\mathrm{Z} \gamma$ process using the ATLAS detector. The study of $\mathrm{W}^{+} \mathrm{W}^{-}$is performed using complementary approaches in ALTAS and CMS. In ATLAS while a zero jet bin is utilized to isolate the $\mathrm{W}^{+} \mathrm{W}^{-}$signal from background processes, in CMS both zero and one jet bins are used. A novel method involving the use of random forest discriminators to suppress backgrounds is also pursued. The $\mathrm{Z} \gamma$ process is studied using the complete Run II dataset of $139 \mathrm{fb}^{-1}$ collected with the ATLAS detector. The fiducial cross section of this process is measured with an unprecedented experimental precision of $2.9 \%$.

The Eighth Annual Conference on Large Hadron Collider Physics-LHCP2020

25-30 May, 2020

online

\footnotetext{
${ }^{*}$ Speaker
} 


\section{Introduction}

The search for dibosons and tribosons offer probes of the non-Abelian $S U(2) \times U(1)$ gauge symmetry of the Standard Model (SM). The study of multiboson physics in the SM spans several orders of magnitude in the production cross section as shown in Fig. 1. The production probabilities can be lower by as much as factors of two (WZ) to 100 (WWW) with respect to Higgs production. In addition to the low production probability, multiboson processes, specifically tribosons, are often difficult to isolate from background processes that have similar features as the signal. This document describes the search for tribosons in leptonic final states (Sec. 2), the precision measurement of diboson processes specifically, $\mathrm{W}^{+} \mathrm{W}^{-}$using both the ATLAS and CMS detectors (Sec. 3 ) and the $\mathrm{Z} \gamma$ process using data from the ATLAS experiment (Sec. 4).
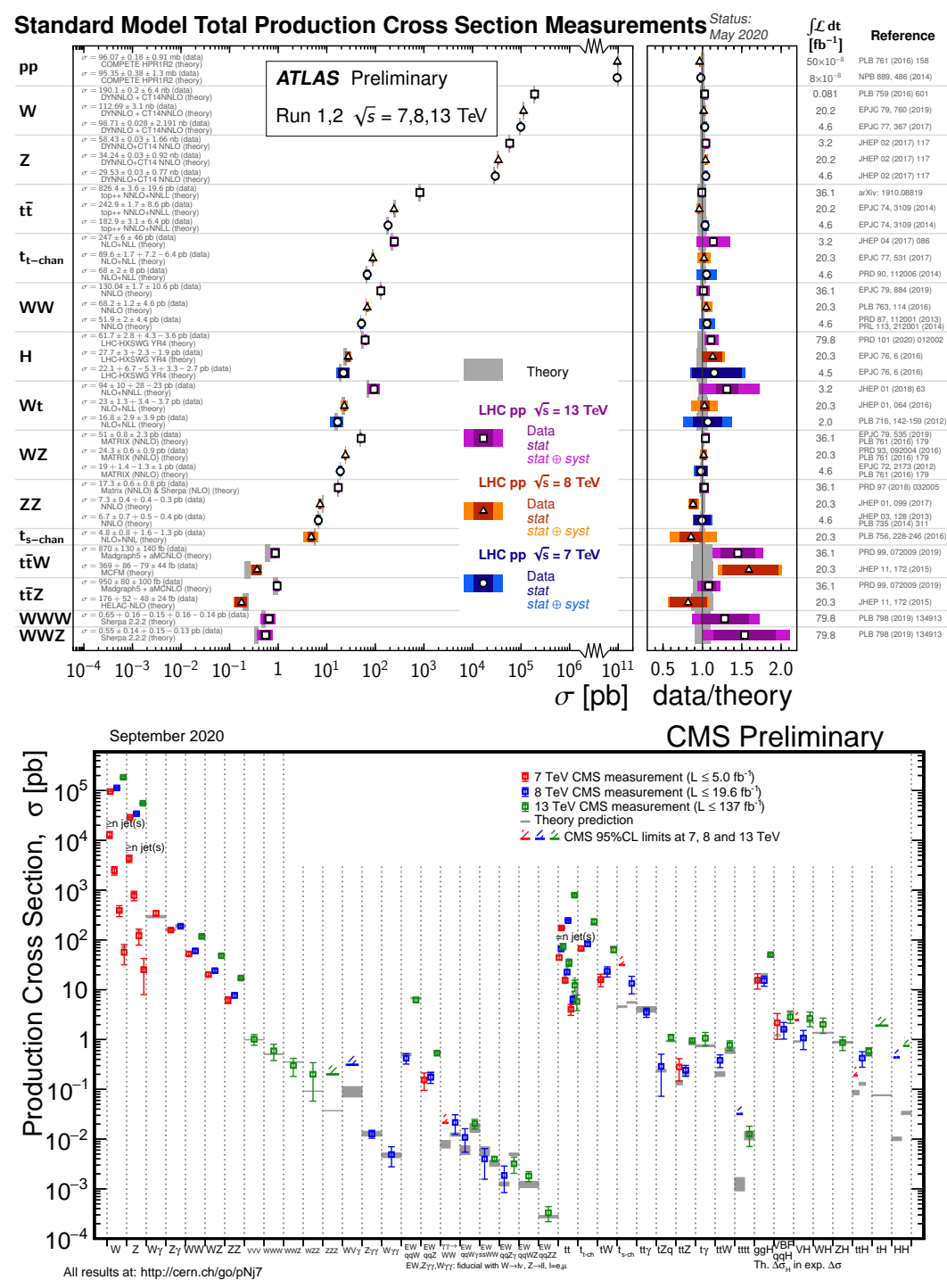

Figure 1: The production cross section of various processes in the SM produced by the ATLAS Collaboration (upper panel) CMS Collaboration (lower panel). 


\section{Search for VVV in leptonic final states [1]}

The production of $\mathrm{VVV}$, where $\mathrm{V}$ is a heavy gauge boson ( $\mathrm{W}$ or $\mathrm{Z}$ ), proceeds through the quartic, trilinear and the Higgs mediated modes. These varied modes of production, as shown in the Feynman diagrams in Fig. 2, make the physics content of these processes illuminating. The first analyses of WWW production were performed by ATLAS [2] and CMS [3] Collaborations at $\sqrt{s}=8$ and $13 \mathrm{TeV}$ respectively. Last year, the ATLAS Collaboration [4] reported the evidence (with an observed (expected) significance of $4.1(3.1) \sigma$ ) of VVV production. This year, the CMS Collaboration reported the first observation of three massive vector boson production at $\sqrt{s}=13$ $\mathrm{TeV}$ with $137 \mathrm{fb}^{-1}$ of data [1] with an observed (expected) significance of 5.7 (5.9) $\sigma$. This marks an important milestone in the physics of multiboson production and is expected to lead to a more detailed analysis of these extremely rare and complex final states. The production cross section of these processes is small and when the leptonic branching fractions are factored in, these rates become smaller as shown in Fig. 3. However, the backgrounds associated with hadronic or semileptonic final states is so large that requiring the presence of multiple leptons with specific charge combination (as in the case of same-signed leptons) becomes imperative. For processes such as WZZ and ZZZ only a few events are expected to be produced at the LHC, signifying the rarity of these processes.

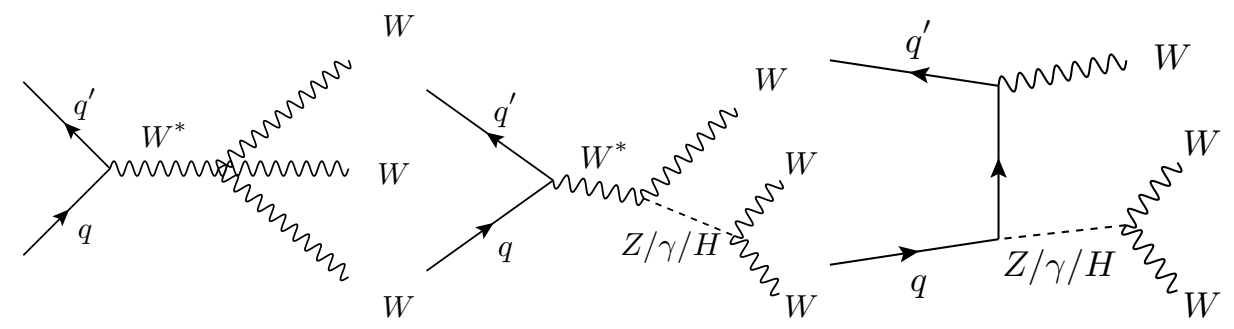

Figure 2: Feynman diagrams associated with the production of WWW. The production proceeds through the quartic, trilinear and the Higgs mediated mode. An additional diagram, not shown here, involves the radiation of three $\mathrm{W}$ bosons from a quark line. The quartic vertex is forbidden in the case of ZZZ production.

The VVV analysis is carried out in two, three, four, five and six lepton channels. The two and three lepton channels are specifically designed to be sensitive to WWW and the four lepton channel is formulated to study the WWZ process, while the five and six lepton channels are directed toward WZZ and ZZZ. Extreme leptonic multiplicities, such as those explored in this analysis, are often unexplored, since signal yields in these channels are low due to the $\mathrm{V} \rightarrow$ leptons branching fractions, which vary between $\sim 22$ and $6 \%$ for $\mathrm{W}$ and $\mathrm{Z}$ bosons respectively. Here leptons refer to electrons and muons. However, five and six lepton multiplicity channels, geared toward the study of WZZ and ZZZ processes, have the advantage of being very "clean", almost devoid of any prompt background.

For channels with large signal and background contributions, several sub-categories are developed to maximize the signal likelihood. In the two, three and four lepton channels a Boosted Decision Tree (BDT) [5] is used to discriminate between signal and backgrounds. In the identically charged dilepton and trilepton categories, two BDTs are trained to mitigate the sources of backgrounds from real leptons and misidentified leptons (labeled nonprompt). The BDT score for the mitigation of nonprompt leptons is shown on the left panel of Fig. 4. The BDTs are trained 


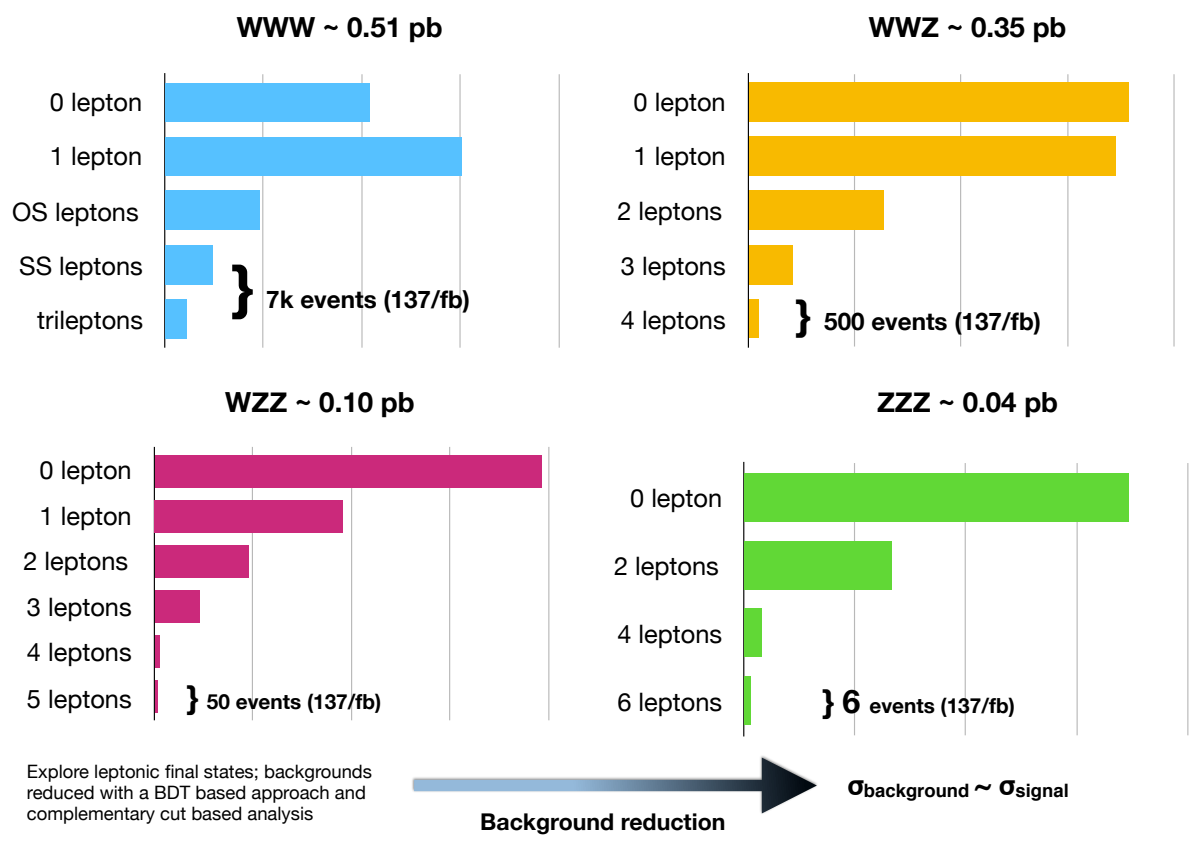

Figure 3: The cross section $\times$ branching fraction for VVV production in various leptonic final states. The number of events expected in $137 \mathrm{fb}^{-1}$ of data is indicated.

with lepton transverse momenta $\left(p_{T}\right)$, jet $p_{T}, E_{T}^{\text {miss }}$ and other kinematic variables. Events with a BDT score $>0.28$ are considered to be part of the signal region in the trilepton final state with a further requirement that no leptons satisfy the same flavor opposite charge criteria. In the four lepton channel, a pair of same flavor opposite charged leptons are required to be consistent with a Z-boson candidate. The invariant mass of the remaining $e \mu$ pair is a powerful discriminant against backgrounds from ZZ, where a $Z$ decays to pairs of $\tau$ leptons (shown on the right panel of Fig. 4) resulting in an invariant mass distribution that is expected to peak near $\sim 50 \mathrm{GeV}$. In the four lepton channel, two BDTs are used, designed to suppress $\mathrm{ZZ}$ and $t \bar{t} \mathrm{Z}$ backgrounds.

\begin{tabular}{|ccc|}
\hline Process & Observed significance $(\sigma)$ & Expected significance $(\sigma)$ \\
\hline WWW & 3.3 & 3.1 \\
WWZ & 3.4 & 4.1 \\
WZZ & 1.7 & 0.7 \\
ZZZ & 0.0 & 0.9 \\
\hline Combined & 5.7 & 5.9 \\
\hline
\end{tabular}

Table 1: Triboson (VVV) production observed for the first time with an observed significance of $5.7 \sigma$. Observed significance for WWW and WWZ at $3.3 \sigma$ and $3.4 \sigma$.

All event categories with signal and background yields in each category can be seen in the upper panel of Fig. 5. There is significant cross channel contamination among various leptonic final states. The VVV signal contributes non-trivially in each of the channels. For example, WWZ signal 

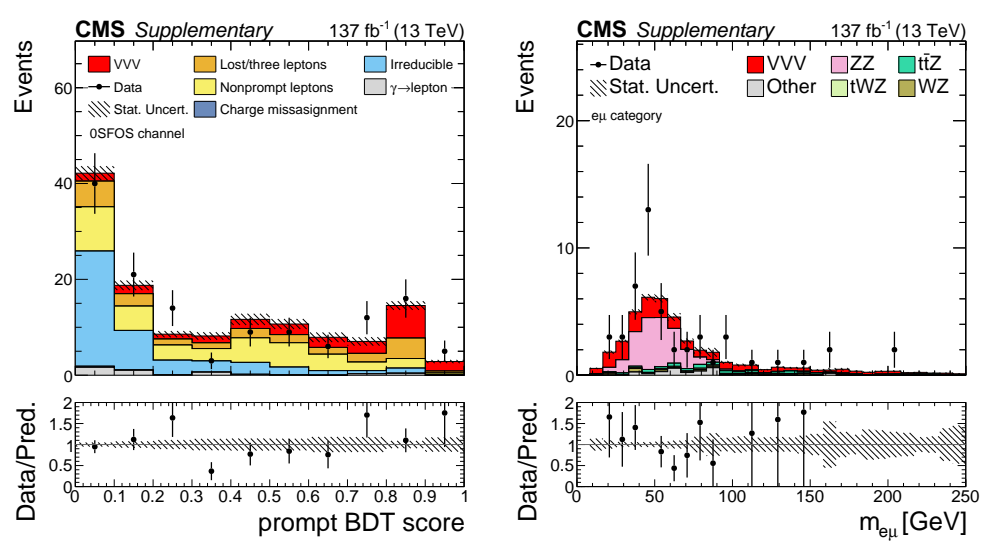

Figure 4: Left: BDT score in the trilepton final state with a further requirement that no leptons satisfy the same flavor opposite charge criteria. Right: The invariant mass of the $e-\mu$ pair in the four lepton region after associating a pair of same flavor opposite charged leptons with a $\mathrm{Z}$ boson candidate.

events are present in the dilepton and trilepton final states, even though those channels are primarily geared toward the WWW process. This necessitates the need for a combined simultaneous analysis of all final states. The observed (expected) combined significance, $\mu_{\text {comb }}$, is 5.7 (5.9) $\sigma$ and the combined signal strength is at $1.02_{-0.23}^{+0.26}$. The significance of WWW and WWZ processes are 3.3 and $3.4 \sigma$ respectively. The expected and observed significances are quoted in Table 1 and shown in the lower panel of Fig. 5. An event display of a spectacular five electron event is shown in Fig. 6.

\section{Precision physics with dibosons: measurement of $\left(\mathrm{W}^{+} \mathrm{W}^{-}\right)$cross section $[6,7]$}

The production of diboson processes, such as $\mathrm{W}^{+} \mathrm{W}^{-}$, at the LHC occurs via $q \bar{q}$ annihilation, the dominant mode (Fig. 7), and via $g g \rightarrow \mathrm{W}^{+} \mathrm{W}^{-}$which involves higher orders in perturbative quantum chromodynamics. Such processes, with cross sections of $\sim 118 \mathrm{pb}$, allow for precision tests of the SM and are therefore pursued in both ALTAS [6] and CMS [7] experiments and involve complementary search strategies in the two experiments. In the ATLAS measurement, the analysis follows a cut based approach performed in a zero jet region, while its CMS counterpart utilizes two methods for background rejection. The cut-based analysis uses both zero and one jet categories, while the second method employs two random forest [8] discriminators to reduce background contributions. The Higgs $\rightarrow \mathrm{W}^{+} \mathrm{W}^{-}$process, with a cross section about ten times smaller than the other process, is considered part of the signal in the ATLAS analysis, while in CMS it is designated as a background.

In the CMS $\mathrm{W}^{+} \mathrm{W}^{-}$analysis, two different complementary search strategies are designed to reduce the top and Drell-Yan backgrounds:

- sequential cut based analysis (in zero and one jet bins)

- random forest classifier trained on simulated events (inclusive in the number of jets)

In both of these approaches same and opposite flavor lepton categories are used. A novel method involves the use of a random forest discriminator is used to distinguish between signal and 


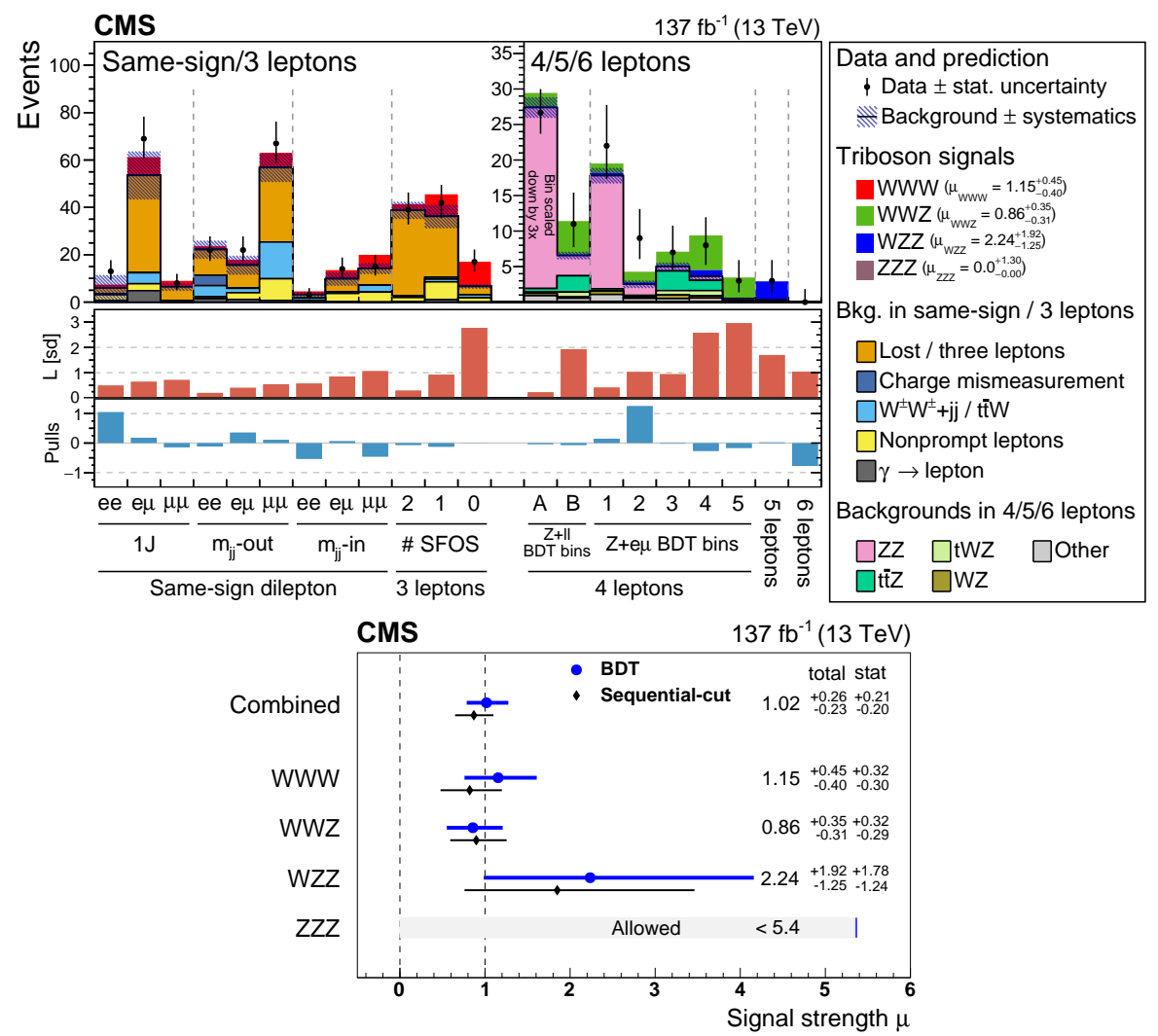

Figure 5: Upper panel: The multichannel exploration of VVV topologies in two, three, four, five and six lepton final states. Lower panel: The observed and expected significances associated with each VVV process.

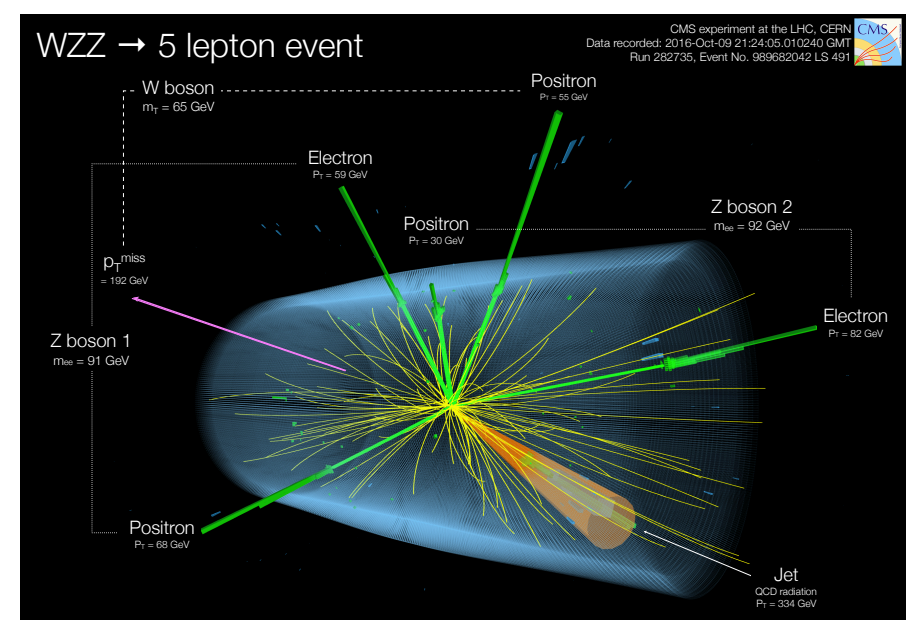

Figure 6: A data event with five leptons that passed all the analysis requirements. The annotation indicates the leptons associated with $\mathrm{W}$ and $\mathrm{Z}$ bosons.

background processes. The discriminator is constructed by independently training a collection of binary decision trees and the score is defined as the combinations of the decision of each tree and is shown in Fig. 8. The features used to train the random forest discriminator is shown in Table 2. 


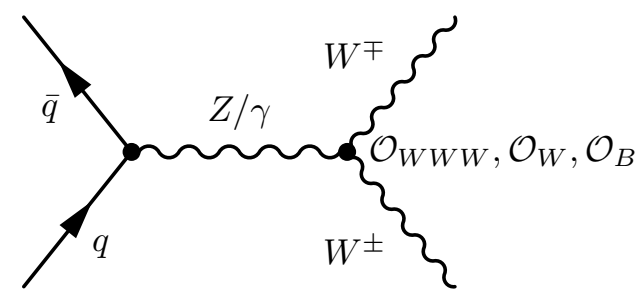

Figure 7: The Feynman diagram showing the s-channel production of $\mathrm{W}^{+} \mathrm{W}^{-}$. The highlighted vertex could acquire contributions from higher-order operators.

This method, unlike other approaches used in both ALTAS and CMS, uses all jet categories.
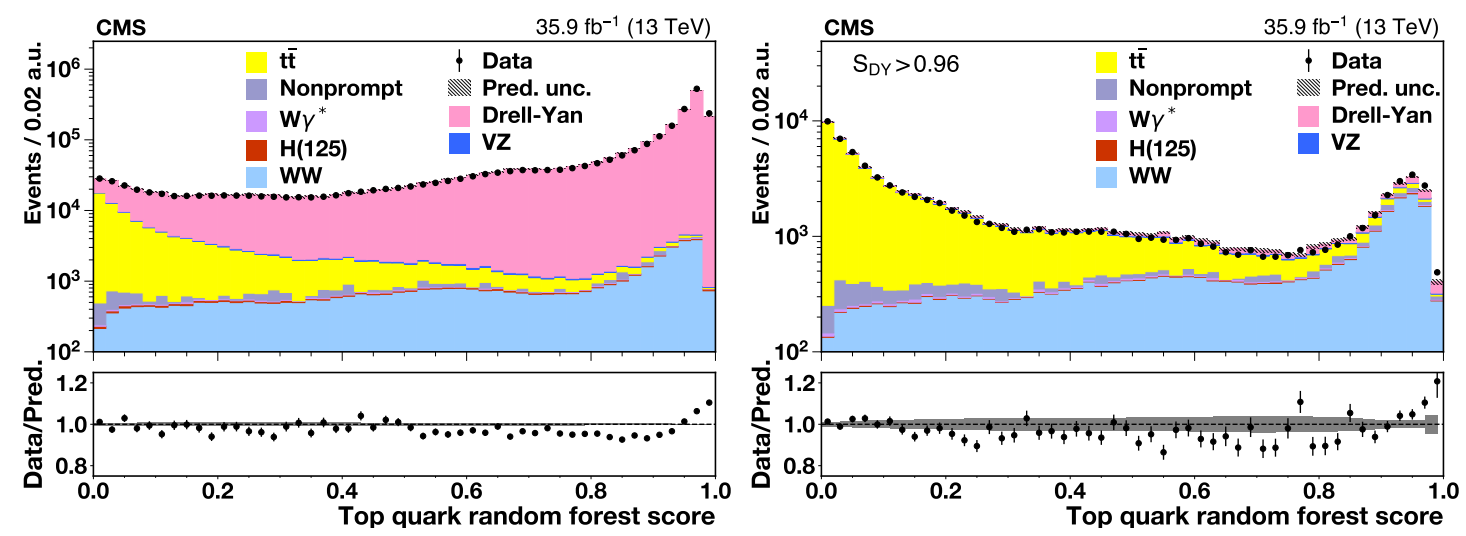

Figure 8: The random forest score for top-like events before (left) and after (center) signal selection requirements (after requiring that the random forest score for Drell-Yan events $>0.96$ ).

The cross section computed is:

$$
\sigma=117.6 \pm 1.4 \text { (stat. }) \pm 5.5 \text { (syst.) } \pm 1.9 \text { (theo.) } \pm 3.2(\text { lumi }) \mathrm{pb}
$$

compared to $\sigma^{\mathrm{NNLO}}=118.8 \pm 3.6 \mathrm{pb}$.

The fraction of events in various jet multiplicity bins with a comparison of the expected number of events from POWHEG is shown on the left panel of Fig. 9. The right panel of Fig. 9 shows the normalized differential cross section as a function of the dilepton mass $m_{\ell \ell}$.

The event selection in the jet binned category in the sequential cut based analysis is sensitive to higher order QCD corrections. The uncertainties associated with the perturbative regime are ascertained by varying factorization and renormalization scales (in some cases $p_{T}$ - resummation techniques are used). Additional sources of theoretical uncertainties include those from the choice of $\alpha_{s}$ and PDFs. The total experimental uncertainties associated with lepton identification and jet energy scale and resolution are at 2.1 and $2.3 \%$ respectively.

The ATLAS analysis exclusively utilizes the $W^{ \pm} W^{\mp} \rightarrow e^{ \pm} v \mu^{\mp} v$ final state in the zero jet bin. The presence of b-jets are vetoed in the event selection. The distribution of the $m_{e \mu}$ invariant mass and the $\left|\cos \theta^{*}\right|$ is shown in Fig. 10.

The total fiducial cross section is computed in a region defined by:

- $p_{T}^{\ell}>27 \mathrm{GeV}$ 


\begin{tabular}{lcc}
\hline \hline Feature & \multicolumn{2}{c}{ Classifier } \\
& Drell Yan & Top quark \\
\hline Lepton flavor & $\checkmark$ & \\
Number of jets & & $\checkmark$ \\
$p_{\mathrm{T}}^{\ell \text { min }}$ & $\checkmark$ & \\
$p_{\mathrm{T}}^{\text {miss }}$ & $\checkmark$ & $\checkmark$ \\
$p_{\mathrm{T}}^{\text {miss,proj }}$ & $\checkmark$ & \\
$p_{\mathrm{T}}^{\ell \ell}$ & $\checkmark$ & $\checkmark$ \\
$m_{\ell \ell}$ & $\checkmark$ & \\
$m_{\ell \ell} p_{\mathrm{T}}^{\text {miss }}$ & $\checkmark$ & \\
$\Delta \phi_{\ell \ell} p_{\mathrm{T}}^{\text {miss }}$ & $\checkmark$ & $\checkmark$ \\
$\Delta \phi_{\ell \mathrm{Jet}}$ & & $\checkmark$ \\
$\Delta \phi_{p_{\mathrm{T}}^{\text {miss }}}$ & & $\checkmark$ \\
$\Delta \phi_{\ell \ell}$ & $\checkmark$ & \\
$H_{\mathrm{T}}$ & $\checkmark$ & $\checkmark$ \\
Recoil & $\checkmark$ & $\checkmark$ \\
\hline
\end{tabular}

Table 2: The variables or features that are given as input to the random forest algorithm. The recoil variable refers to the vectorial sum of the jet momenta.
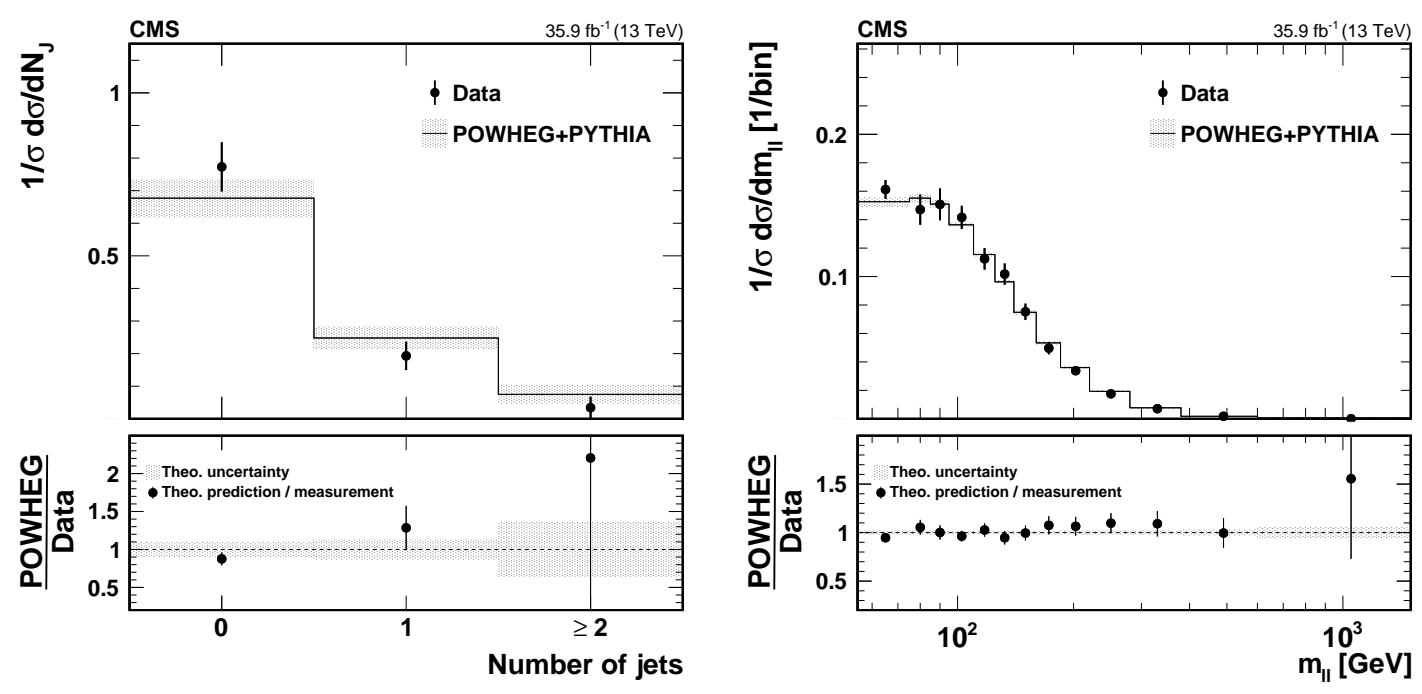

Figure 9: The left panel shows the fiducial cross section as a function of the number of jets and the right panel shows the fiducial cross section as a function of invariant mass of the two leptons $m_{l l}$. This variable is expected to be sensitive to the presence of higher order operators.

- $\left|\eta^{\ell}\right|<2.5$

- $m_{e \mu}>55 \mathrm{GeV}$

- $p_{\mathrm{T}}^{e \mu}>30 \mathrm{GeV}$

- $E_{\mathrm{T}}^{\mathrm{miss}}>20 \mathrm{GeV}$

- No jets with $p_{\mathrm{T}}>35 \mathrm{GeV},|\eta|<4.5$ 
The measured value of the fiducial cross section is

$$
\left.\left.\sigma_{\text {fid }}=379.1 \pm 5.0 \text { (stat. }\right) \pm 25.4 \text { (syst. }\right) \pm 8.0(\text { lumi }) \mathrm{fb}
$$

The largest sources of systematic uncertainties are associated with the $b$-tagging (3.4\%), jet energy scale $(3 \%)$, top quark $(2.6 \%)$ and the $\mathrm{W}+$ jets $(3.1 \%)$ modeling.
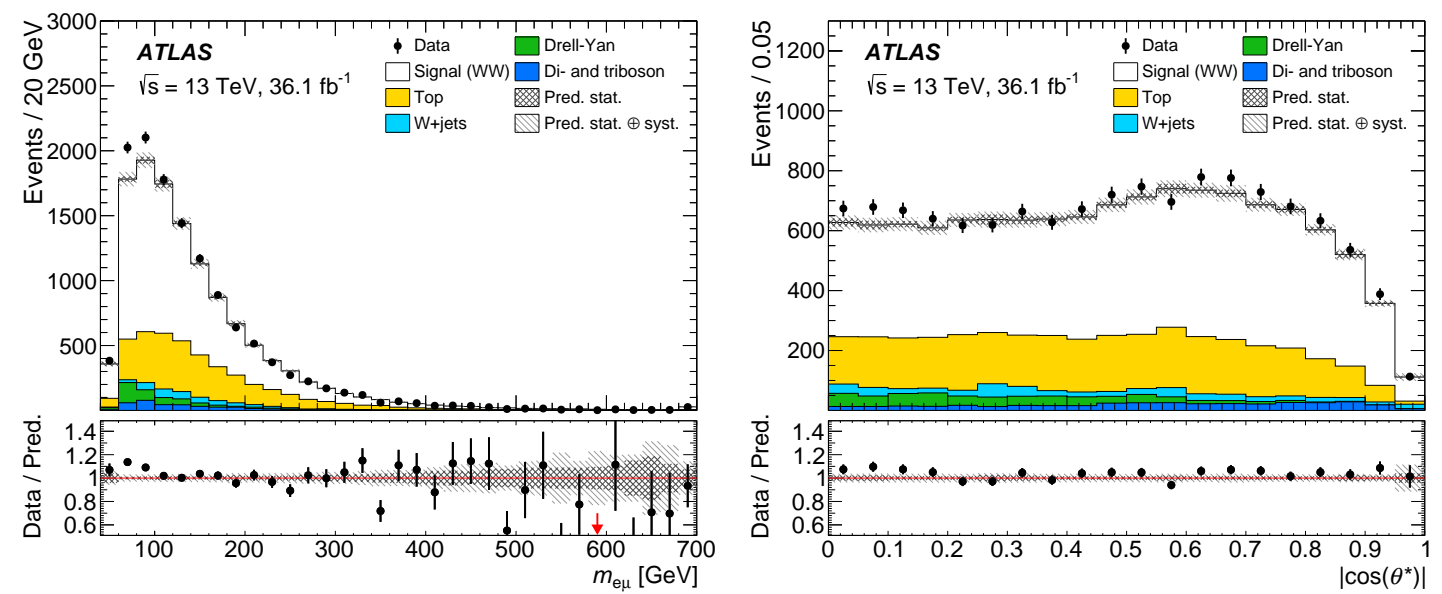

Figure 10: The left panel shows the $m_{e \mu}$ invariant mass spectrum. The right panel shows the $\left|\cos \theta^{*}\right|=$ $\left|\tanh \left(\frac{\Delta \eta_{e \mu}}{2}\right)\right|$, a variable that is sensitive to the spin structure of $e \mu$ pairs.

\subsection{Limits on dimension-6 Wilson Coefficients}

The SM Lagrangian is based on operators of dimension-4. The production of $\mathrm{W}^{+} \mathrm{W}^{-}$involves various vertices which could acquire contributions from higher dimensional operators. The Effective Field Theory (EFT) framework operates under the assumption that new physics exists at scales currently inaccessible at the LHC, but whose indirect effects can be detected as excesses of events in the tails of distributions. The EFT framework extends the SM Lagrangian in an expansion in inverse powers of the scale of new physics as shown in Eqn. 3.

$$
\mathcal{L}=\mathcal{L}_{\mathrm{SM}}+\sum_{i} \frac{c_{i}}{\Lambda^{2}} O_{i}+\sum_{j} \frac{f_{j}}{\Lambda^{4}} O_{j}+\ldots
$$

The following CP-conserving dimension-6 operators are included in the non-SM part of the Lagrangian [9]:

$$
\begin{aligned}
O_{\mathrm{WWW}} & =\operatorname{Tr}\left[W_{\mu \nu} W^{\nu \rho} W_{\rho}^{\mu}\right] \\
O_{\mathrm{B}} & =\left(D_{\mu} \Phi\right)^{\dagger} B^{\mu \nu}\left(D_{\nu} \Phi\right) \\
O_{\mathrm{W}} & =\left(D_{\mu} \Phi\right)^{\dagger} W^{\mu \nu}\left(D_{\nu} \Phi\right)
\end{aligned}
$$

The $\mathrm{CP}$ violating operators are of the following form:

$$
\begin{aligned}
O_{\tilde{\mathrm{W}} \mathrm{WW}} & =\operatorname{Tr}\left[\tilde{W}_{\mu \nu} W^{\nu \rho} W_{\rho}^{\mu}\right] \\
O_{\tilde{\mathrm{W}}} & =\left(D_{\mu} \Phi\right)^{\dagger} \tilde{W}^{\mu \nu}\left(D_{\nu} \Phi\right)
\end{aligned}
$$

The observed and expected limits associated with the Wilson coefficients computed in both the ATLAS and the CMS analyses are shown in Table 3. 
ATLAS

CMS

Coupling Observed allowed range Expected range Observed allowed range Expected range

\begin{tabular}{|ccccc|} 
& $\left(\mathrm{TeV}^{-2}\right)$ & $\left(\mathrm{TeV}^{-2}\right)$ & $\left(\mathrm{TeV}^{-2}\right)$ & $\left(\mathrm{TeV}^{-2}\right)$ \\
\hline$c_{\mathrm{WWW}} / \Lambda^{2}$ & {$[-3.4,3.3]$} & {$[-3.0,3.0]$} & {$[-1.8,1.8]$} & {$[-2.7,2.7]$} \\
$c_{\mathrm{W}} / \Lambda^{2}$ & {$[-7.4,4.1]$} & {$[-6.4,5.1]$} & {$[-3.6,2.8]$} & {$[-5.3,4.2]$} \\
$c_{\mathrm{B}} / \Lambda^{2}$ & {$[-21,18]$} & {$[-18,17]$} & {$[-9.4,8.5]$} & {$[-14,13]$} \\
$c_{\tilde{\mathrm{WWW}}} / \Lambda^{2}$ & {$[-1.6,1.6]$} & {$[-1.5,1.5]$} & & \\
$c_{\tilde{\mathrm{W}}} / \Lambda^{2}$ & {$[-76,76]$} & {$[-91,91]$} & & \\
\hline
\end{tabular}

Table 3: The 95\% CL limits on the Wilson coefficients associated with dimension- 6 operators.

\section{Precision Physics with dibosons: measurement of $(\mathrm{Z} \gamma)$ cross section [10]}

The production of $Z$ boson in association with a photon $(\gamma)$ offers an important probe of the electroweak sector of the SM. This process is also an important background for cases where the Higgs boson decays to the $Z \gamma$ final state. The $Z \gamma$ process is characterized by the presence of a photon associated with initial state radiation (left panel of Fig. 11), while the background process features a photon arising out of final state radiation (FSR) as shown in the center panel of Fig. 11. Photons arising from FSR are suppressed by placing stringent requirements on the sum of the two-body (invariant mass of the two leptons, $m_{\ell \ell}, \ell=e, \mu$ ) and the three-body (invariant mass of the dilepton and the photon system, $m_{\ell \ell \gamma}$ ) mass (Fig. 11). The previous exploration of this final state focused on photon transverse energy, $E_{T}>150 \mathrm{GeV}$ and $175 \mathrm{GeV}$ in the $Z \gamma \rightarrow v v \gamma$ [11] and $Z \gamma \rightarrow b b \gamma$ [12] final states respectively, where the goal was to be sensitive to the presence of higher dimensional operators. This analysis explores a wide $E_{T}$ range of $30-1200 \mathrm{GeV}$ and provides a highly precise measurement of the fiducial cross section.
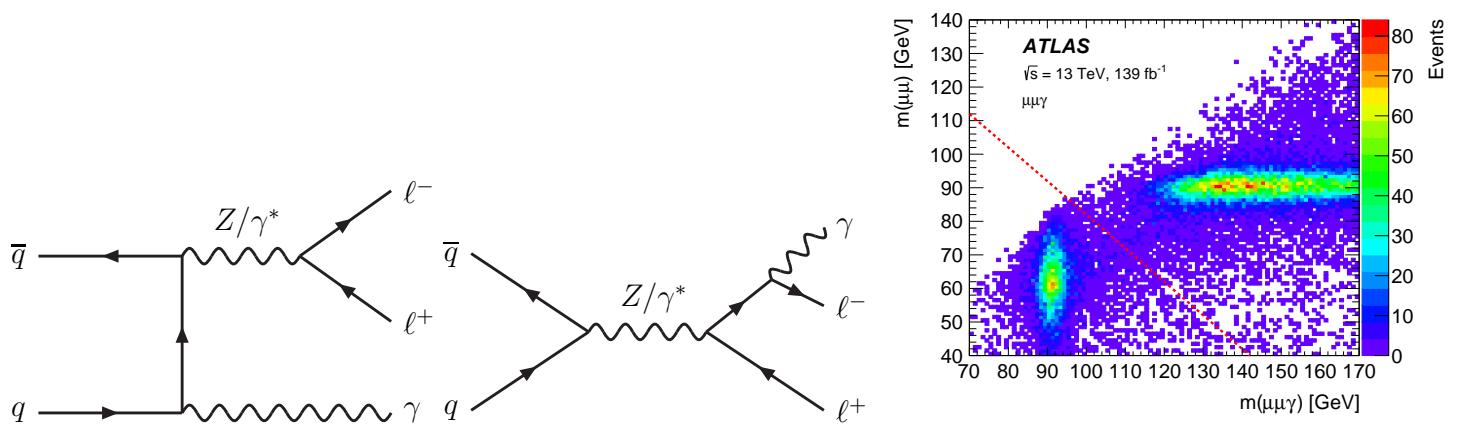

Figure 11: The left panel shows the signal process where the photon originates from initial state radiation. The center panel shows a background process where the photon is from the final state radiation and is most likely to mimic the signal. The right panel shows the distribution of the two-body versus the three body mass, where the requirement of $m_{\ell \ell}+m_{\ell \ell \gamma}>182 \mathrm{GeV}$ is deemed sufficient to reduce FSR contamination.

The backgrounds that contribute to the $(Z \rightarrow \ell \ell) \gamma$ final state include $Z+j$ jets, where a jet is misidentified as a photon. This is by far the dominant background contribution. Other backgrounds are associated with top-quark or multiboson processes. Another source of background is from pile- 
up, where the selected photon and the lepton pair originate from different $p p$ interactions within the same LHC bunch crossing. The $Z+$ jets background is estimated using the sidebands [13] that factor in the probability of a jet passing the photon identification and isolation requirement. The estimation of this background is then validated in three control regions defined by modifying either the photon isolation or identification requirements or both. The background arising from pile-up interactions is estimated by exploiting the fact that there is no correlation between the $z$-positions of the $Z$-boson and the photon for these events. This feature is in sharp contrast with the signal arising from the hard interaction. All other sources of backgrounds are estimated using Monte Carlo simulations.

The signal region is defined requiring $m_{\ell \ell}>40 \mathrm{GeV}$ in addition to the FSR suppression. The distribution of the $\mathrm{m}_{\ell \ell \gamma}$ and $E_{\mathrm{T}}^{\gamma}$ in the signal region can be seen in Fig. 12, where the excellent agreement between data and simulations extends all the way from 30 to $1200 \mathrm{GeV}$ in the $E_{T}$ spectra. While the plots here show the $e^{+} e^{-} \gamma$ signal region, similar behavior is observed in the $\mu^{+} \mu^{-} \gamma$ region as well.
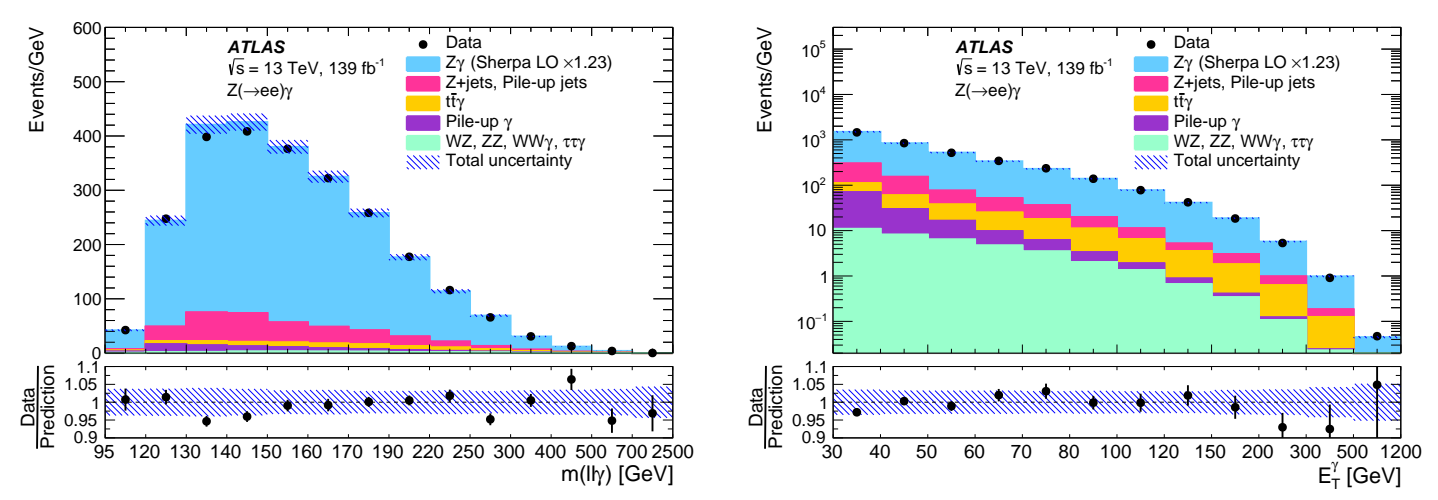

Figure 12: The distribution of the $\mathrm{m}_{\ell \ell \gamma}$ (left) and $E_{\mathrm{T}}^{\gamma}$ (right) in the signal region, specifically $Z \rightarrow e^{+} e^{-} \gamma$. The signal process simulated with SHERPA [14] at leading order, represented in the above plots as a blue filled histogram, includes a normalization factor of 1.23 .

The fiducial cross section is computed in a region similar to the signal region with additional particle-level requirements on the isolation of photons and the lepton four-momenta are corrected by a procedure known as "dressing" [10]. The particle-level quantities are defined with respect to the stable particles in the Monte Carlo simulation. The fiducial cross section as a function of the $E_{\mathrm{T}}^{\gamma}$ and the $\mathrm{m}_{\ell \ell}$ spectra is shown in Fig. 13 and is calculated as:

$$
\sigma_{\text {fid }}=\frac{N_{\text {obs }}-N_{\text {bkg }}}{C \times \mathcal{L}}
$$

where $N_{\text {obs }}$ is the observed number of selected data events in the signal region, $N_{\mathrm{bkg}}$ is the expected number of background events, $\mathcal{L}$ is the integrated luminosity and the effect of detection efficiency and acceptance is factored into $C$. This correction factor $C$ is determined using SHERPA 2.2.4 [14] at LO in the signal region.

Systematic uncertainties in the measured cross section originate from uncertainties in $C, N_{\mathrm{bkg}}$, $\mathcal{L}$ and the unfolding procedure. Uncertainties associated with $C$ include trigger, reconstruction, 

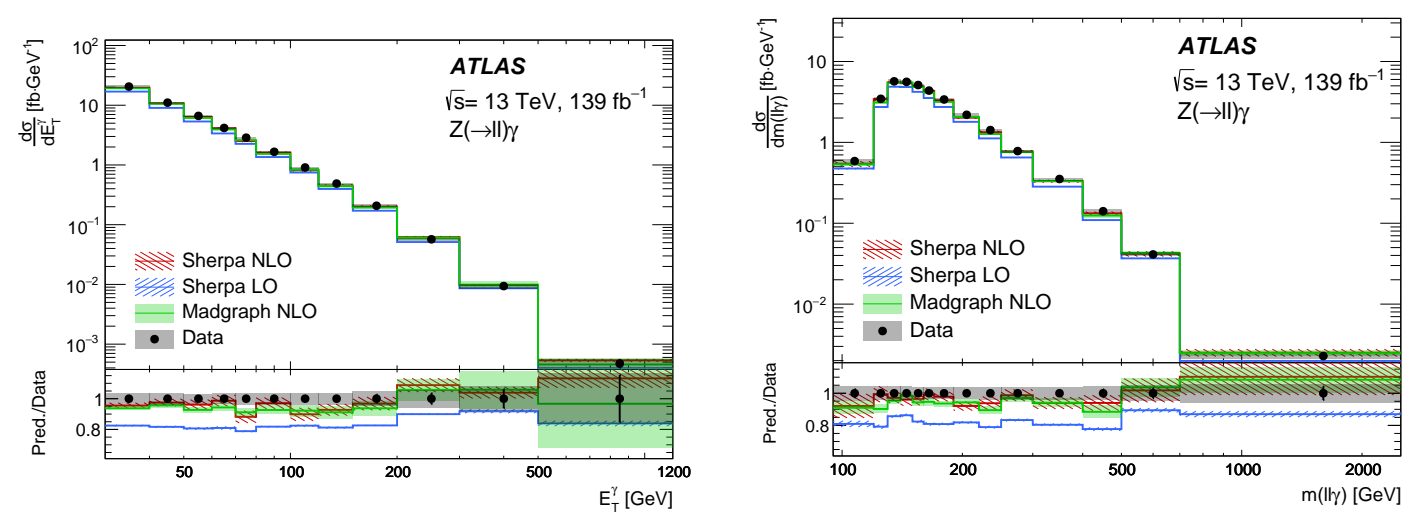

Figure 13: The fiducial cross section as a function of the $E_{\mathrm{T}}^{\gamma}$ (left) and the $\mathrm{m}_{\ell \ell \gamma}$ (right) spectra. The measured cross sections are compared with the SM expectations computed using SHERPA LO [14], NLO [15] and MADGRAPH5_aMC@NLO [16] event generators at particle-level.

particle identification and isolation uncertainties and energy and momentum scale and resolution uncertainties of reconstructed photons, electrons and muons. The total measured cross section for $\ell^{+} \ell^{-} \gamma(\ell=e, \mu)$ production is

$$
\sigma_{\text {fid }}=533.7 \pm 2.1 \text { (stat.) } \pm 12.4 \text { (stat.) } \pm 9.1 \text { (lumi.) fb }
$$

The relative uncertainty of the cross section measurement is $2.9 \%$ and represents a highly precise measurement of the cross section of $Z(\rightarrow \ell \ell) \gamma$ process.

\section{Conclusion}

The diboson and triboson searches presented in this document are representative of the various advances in the field of multiboson physics. With the availability of larger datasets in the High Luminosity LHC era, processes such as triboson production can be studied in great detail elucidating the non-Abelian gauge structure of the SM. In the realm of diboson physics, highly precise measurements of cross sections are expected to drive theoretical advancements in the computation of higher order corrections and the reduction of uncertainties associated with the perturbative regime of quantum chromodynamics.

\section{References}

[1] CMS Collaboration, Observation of heavy triboson production in leptonic final states in proton-proton collisions at $\sqrt{s}=13 \mathrm{TeV}$, Phys. Rev. Lett. 125 (2020) 151802, arXiv:2006.11191 [hep-ex]

[2] ATLAS Collaboration, Search for triboson $W^{ \pm} W^{ \pm} W^{\mp}$ production in $p p$ collisions at $\sqrt{s}=8$ TeV with the ATLAS detector, Eur. Phys. J. C 77 (2017) 141, arXiv:1610.05088 [hep-ex]

[3] CMS Collaboration, Search for the production of $W^{ \pm} W^{ \pm} W^{\mp}$ events at $\sqrt{s}=13 \mathrm{TeV}$, Phys. Rev. D 100, 012004 (2019), arXiv:1905.04246 [hep-ex] 
[4] ATLAS Collaboration, Evidence for the production of three massive vector bosons with the ATLAS detector, arXiv:1903.10415 [hep-ex]

[5] Byron P. Roe et al., Boosted decision trees as an alternative to artificial neural networks for particle identification, NIM A: Volume 543, Issues 2-3 (2005), Pages 577-584

[6] ATLAS Collaboration, Measurement of fiducial and differential $\mathrm{W}^{+} \mathrm{W}^{-}$production crosssections at $\sqrt{s}=13$ with the ATLAS detector, Eur. Phys. J. C 79 (2019) 884, arXiv:1905.04242 [hep-ex]

[7] CMS Collaboration, Studies of $\mathrm{W}^{+} \mathrm{W}^{-}$production in proton-proton collisions at $\sqrt{s}=$ $13 \mathrm{TeV}$, arXiv:2009.00119 [hep-ex]

[8] L. Breiman, Random Forests, Mach. Learn. 45, 5 (2001)

[9] C. Degrande et al., Effective Field Theory: A Modern Approach to Anomalous Couplings, Annals of Physics, Volume 335, August 2013, Pages 21-32, arXiv:1205.4231 [hep-ph]

[10] ATLAS Collaboration, Measurement of the $Z \rightarrow l^{+} l^{-} \gamma$ production cross-section in pp collisions at $\sqrt{s}=13 \mathrm{TeV}$ with the ATLAS detector, Journal of High Energy Physics, JHEP 03 (2020) 054, arXiv:1911.04813 [hep-ex]

[11] ATLAS Collaboration, Measurement of the $Z \gamma \rightarrow v \bar{v} \gamma$ production cross section in $p p$ collisions at $\sqrt{s}=13 \mathrm{TeV}$ with the ATLAS detector and limits on anomalous triple gaugeboson couplings, JHEP 12 (2018) 010, arXiv:1801.04995 [hep-ex]

[12] ATLAS Collaboration, Measurement of the jet mass in high transverse momentum $Z(\rightarrow b \bar{b}) \gamma$ production at $\sqrt{s}=13 \mathrm{TeV}$ using the ATLAS detector, (2019), arXiv:1907.07093 [hep-ex]

[13] ATLAS Collaboration, Measurement of the inclusive isolated prompt photon cross section in pp collisions at $\sqrt{s}=8 \mathrm{TeV}$ with the ATLAS detector, JHEP 08 (2016) 005, arXiv: 1605.03495 [hep-ex]

[14] SHERPA collaboration, Event generation with Sherpa 2.2, SciPost Phys.7 (2019) 034

[15] J. Krause and F. Siegert, NLO QCD predictions for $Z+\gamma+$ jets production with Sherpa, Eur. Phys. J. C 78 (2018) 161, arXiv: 1708.06283 [hep-ph]

[16] J. Alwall et al., The automated computation of tree-level and next-to-leading order differential cross sections, and their matching to parton shower simulations, J. High Energy Phys. 2014, 079 